\title{
Biomasa aérea y ecuaciones alométricas en un cafetal en la Sierra Norte de Oaxaca
}

\section{Aerial biomass and allometric equations in a coffee plantation in the Sierra Norte de Oaxaca}

\author{
Mayra Atalí Terán-Ramírez ${ }^{1}$, Gerardo Rodríguez-Ortiz ${ }^{1 *}$, José Raymundo Enríquez-del Valle ${ }^{1}$, Vicente \\ Arturo Velasco-Velasco ${ }^{1}$ \\ ${ }^{1}$ División de Estudios de Posgrado e Investigación, Instituto Tecnológico del Valle de Oaxaca. Ex-Hda. de Nazareno S/N, CP. \\ 71233, Santa Cruz Xoxocotlán, Oaxaca, México \\ *Autor de correspondencia: grodriguez.itvo@yahoo.com
}

Artículo científico recibido: 02 de mayo de 2017 aceptado: 19 de enero de 2018

RESUMEN. Los sistemas agroforestales de café (SAFC) son de importancia productiva en el estado de Oaxaca. En los últimos 20 años han sido afectados por diversos factores que promueven el cambio de uso de suelo e incrementan los gases de efecto invernadero. Con el objetivo de analizar la estructura y acumulación de biomasa aérea en un SAFC y ajustar ecuaciones alométricas de biomasa para Coffea arabica L., se estableció un experimento bajo un diseño completamente al azar, con tres niveles de cobertura de copa en unidades experimentales de $400 \mathrm{~m}^{2}$, en las que se registraron medidas dasométricas de todos los árboles, los datos se sometieron a un análisis de varianza y pruebas de medias de Duncan $(\mathrm{p} \leq 0.05)$; por medio de regresión se seleccionaron las ecuaciones de biomasa aérea de los cafetos. La estructura del SAFC es irregular, se identificaron 14 especies presentando mayor frecuencia las especies Inga edulis Mart (749 árboles ha ${ }^{-1}$ ) y Liquidambar styraciflua L. (493 árboles ha ${ }^{-1}$ ). La mayor acumulación de biomasa se encontró en SAFC con nivel medio de cobertura para árboles sombra y total aéreo de 43 y $86 \%$ con $78.4 \pm 29.4$ y 87.02 $\pm 33.3 \mathrm{t} \mathrm{ha}^{-1}$. La biomasa en el piso no mostró diferencias significativas entre los niveles de cobertura evaluados. Los compartimentos de biomasa aérea para $C$. arabica se estiman con coeficientes de ajuste entre 0.71 y 0.89 , donde el diámetro basal y altura de los cafetos son variables independientes.

Palabras clave: Cobertura de copa, Coffea arabica L., ecuaciones alométricas, estructura horizontal, estructura vertical

ABSTRACT. Coffee agroforestry systems (CAFS) are of productive importance in the state of Oaxaca. In the last 20 years they have been affected by various factors that promote land-use change and thereby increase greenhouse gases. In order to analyze the structure and accumulation of aerial biomass in a CAFS and fit allometric biomass equations for Coffea arabica L., an experiment was established under a completely randomized design, under three levels of crown cover in $400 \mathrm{~m}^{2}$ experimental units in which dasometric measurements of all the trees were recorded; the data were subjected to an analysis of variance and Duncan's multiple range test $(p \leq 0.05)$. By means of regression, the aerial biomass equations of the coffee trees were selected. The CAFS structure is irregular; in total, 14 species were identified, with the most frequently occurring being the species Inga edulis Mart (749 trees ha ${ }^{-1}$ ) and Liquidambar styraciflua L. (493 trees ha ${ }^{-1}$ ). The highest biomass accumulation was found in CAFS with mean cover level for shade trees and aerial total of 43 and $86 \%$ with $78.4 \pm 29.4$ and $87.02 \pm 33.3 \mathrm{t} \mathrm{ha}^{-1}$. The biomass on the ground did not show significant differences among the cover levels evaluated. The aerial biomass compartments for $C$. arabica are estimated with fit coefficients between 0.71 and 0.89 , where the basal diameter and height of the coffee trees are independent variables.

Key words: Crown cover, Coffea arabica L., allometric equations, horizontal structure, vertical structure 


\section{INTRODUCCIÓN}

La reducción de emisión de gases de efecto invernadero (GEI), es una necesidad prioritaria para mitigar los impactos del calentamiento global (IPCC 2007). Una opción es conservar los recursos naturales, debido a que las plantas mediante la fotosíntesis retienen y almacenan $\mathrm{CO}_{2}$ en su biomasa, por lo que una alternativa es el establecimiento de sistemas agroforestales (SAF), en los que se establecen cultivos con árboles y/o ganadería, con plantas perennes con alto potencial de almacenamiento de biomasa y carbono (C) (Ramachandran y Nair 2014). Para determinar la capacidad de almacenamiento de $\mathrm{C}$ de estos sistemas, es necesario realizar estudios de biomasa, debido a que se encuentra en función de factores físicos y ambientales (Soto-Pinto et al. 2009). Se estima que entre el 40 y $50 \%$ de la biomasa corresponde a C (IPCC 2007), lo que permite ingresar a los Mecanismos de Desarrollo Limpio mediante bonos de C (BenjamínOrdoñez y Masera 2001). Las prácticas de la agricultura climática inteligente, tienen un enfoque que permite satisfacer las necesidades alimentarias de la población, que se adapten y sean resilientes a los cambios climáticos y que al mismo tiempo ayude a mitigar los efectos del cambio climático (Ramachandran y Nair 2014). Dentro de estas prácticas se encuentran los SAF, que proporcionan servicios ambientales como conservación y retención de suelos, captura y mejora en la calidad de agua, belleza escénica y conservación de la biodiversidad (Ramachandran 2011).

En México, predominan los cultivos de café bajo la sombra del dosel de bosques y selvas, conocidos como sistemas agroforestales de café (SAFC), en un esquema de cultivo orgánico de acuerdo con los principios de producción sustentable, alternativa viable para mejorar las condiciones de vida de los campesinos que se dedican a esta actividad (Moguel y Toledo 1999). Los árboles sombra en estos SAFC tienen influencia sobre el crecimiento de los cafetales, debido a que requieren intensidades de radiación solar bajas para realizar la fotosíntesis (Montagnini et al. 2015) y promover la fijación de nu- trientes (Negash et al. 2013). Un SAFC, con adecuado nivel de sombra tiene equilibrio de la humedad y evita la proliferación de plagas y enfermedades (Maldonado-Martínez et al. 2014).

La estructura del SAFC puede analizarse por su diversidad de especies, el número de individuos por especie y su distribución espacial (Escamilla et al. 1997). La biodiversidad en los sistemas agroforestales es el centro de atención de muchos investigadores que destacan la importancia de los SAFC para conservar la diversidad arbórea y la estructura del sistema (Valencia et al. 2014). Al respecto García et al. (2015) estudiaron la estructura y biodiversidad arbórea en un SAFC, con índices de biodiversidad, determinando mayor índice de diversidad y de importancia en policultivos con Cordia alliadora Ruiz y Pav. Los SAF se componen de múltiples capas de dosel, las cuales son útiles para la conservación de suelos y la retención de agua, como lo demostraron Pérez et al. (2012) quienes indican una relación negativa entre la cobertura y la de pérdida de suelos.

Debido a la compleja estructura de los SAF estos acumulan biomasa en todos sus niveles, lo que se traduce en mayor captura de C (Häger 2012). El contenido de $\mathrm{C}$ de la biomasa acumulada en los SAFC, tiene un factor de conversión de 0.5 sobre la biomasa de Coffea arabica L., con Cedrela odorata L., Macadamia integrifolia Maiden, M. integrifolia Maiden y Musa paradisiaca L., con captura promedio de $102 \mathrm{t} \mathrm{C} \mathrm{ha}^{-1}$ (Espinoza-Domínguez et al. 2012). Mientras que Andrade et al. (2014) reportan que la tasa de fijación anual de $C$ es de 0.6 a 4.37 $\mathrm{t}$ ha ${ }^{-1}$ en cultivos de café bajo sol. En los SAFC con Cordia alliadora Ruiz y Pav. y Hevea brasiliensis Willd; se tiene una captura de $14 \mathrm{t} \mathrm{C} \mathrm{ha}^{-1}$ en la parte aérea (Magaña et al. 2004). Para determinar el contenido de $\mathrm{C}$, se han construido ecuaciones alométricas para calcular la biomasa acumulada en SAF con café (Segura et al. 2006, Negash et al. 2013).

En la localidad de San Juan Juquila Vijanos se tiene la necesidad de promover la conservación de los sistemas agroforestales de café orgánico, debido a los bajos ingresos de la producción de café y la 
ocurrencia de plagas. Lo que ha disminuido la superficie, debido al desmonte para establecer cultivos anuales y las altas pendientes de la zona, se estima la pérdida de $48.5 \mathrm{t} \mathrm{ha}^{-1}$ año $^{-1}$ de suelo (Santos 2012). Por lo anterior, el objetivo del estudio fue determinar la relación entre la estructura y la acumulación de biomasa aérea del sistema agroforestal de café orgánico y desarrollar ecuaciones alométricas de biomasa aérea de $C$. arabica $\mathrm{L}$.

\section{MATERIALES Y MÉTODOS}

\section{Área de estudio}

Se realizó en un área de 1.2 ha, en la localidad San Juan Juquila Vijanos, en la Sierra Norte del estado de Oaxaca, en las coordenadas $17^{\circ} 18^{\prime}$ y $17^{\circ} 23^{\prime} \mathrm{LN}$ y $96^{\circ} 14^{\prime}$ y $96^{\circ} 20^{\prime} \mathrm{LO}$; con altitudes entre 900 y $2400 \mathrm{~m}$. Los terrenos presentan pendientes del 25 al $60 \%$. El clima predominante es semicálido húmedo con abundantes lluvias en verano, precipitación entre 1200 y 2000 mm; con temperatura media anual entre 16 y $22{ }^{\circ} \mathrm{C}$ (INEGI 2006). Las parcelas de estudio se encuentran en un sistema agroforestal de café con sombra en policultivo bajo producción orgánica, con elementos que forman el bosque mesófilo de montaña con especies nativas e introducidas, en terrenos con pendientes menores a $40 \%$ y altitud entre 1200 y 1600 m.

\section{Establecimiento de sitios experimentales}

El experimento se estableció bajo un diseño completamente al azar, utilizando como tratamientos tres niveles de cobertura de copa bajo $(<43 \%)$, medio $(43-86 \%)$ y alto $(>86 \%)$ con base en la metodología reportada por Maldonado-Martínez et al. (2014), ajustando los valores de acuerdo a la densidad de árboles sombra promedio (75, 86 y 112 árboles $\mathrm{ha}^{-1}$, respectivamente). Se establecieron nueve sitios de muestreo circulares de $400 \mathrm{~m}^{2}$, en los que se realizó el inventario del arbolado midiendo el diámetro al pie $(\mathrm{db})$ y diámetro normal $(\mathrm{dn})$ en $\mathrm{cm}$ a $1.3 \mathrm{~m}$ de alto con una cinta diamétrica; altura (at) en metros con un dendrómetro (modelo Haga); diámetro de copa (dcop) en metros con cinta métrica, en los cafetos se midieron con cinta métrica la at, db y dcop. Para el arbolado sombra y cafetos utilizando el dcop se calculó el área de copa (acop) con la fórmula acop $=\frac{\pi}{4} x d c o p^{2}$ (en $\mathrm{m}^{2}$ ) para establecer los tratamientos mediante la fórmula: $c o b=\frac{\sum a c o p}{a r e a U E} \times 100$. Donde $c o b=$ porcentaje de cobertura de árboles sombra, acop = área de copa de árboles sombra, area $U E=$ área de la unidad experimental.

Posteriormente se derribaron 30 cafetos cubriendo todos los tamaños existentes en la parcela; para luego separarlos en hojas, ramas y fuste, para determinar su peso fresco (PF) en gramos en campo con una balanza electrónica (CS500-0W0). De cada compartimento se tomó una muestra, la cual se pesó en fresco con una balanza electrónica en campo y se colocó en bolsas de papel y se llevó al laboratorio; para secarla en una estufa marca Memmert (Modelo 100-800) a $80{ }^{\circ} \mathrm{C}$ hasta obtener peso constante. Las muestras secas se pesaron en una balanza analítica Shimadzu (ATY224, Japón) para determinar el peso seco (PS) en gramos, para luego obtener un factor de conversión por muestra con la formula $\mathrm{FC}=\mathrm{PF} / \mathrm{PS}$ Donde: $F C=$ factor de conversión, $P S=$ peso seco y $\mathrm{PF}=$ peso fresco. Con el valor de FC por muestra se determinó la biomasa total por compartimento.

Dentro de cada sitio se estableció un sub-sitio cuadrado de $25 \mathrm{~m}^{2}$ en el que se recolectó y pesó la necromasa (ramillas con diámetro mayor a $5 \mathrm{~cm}$ ) en gramos en una balanza Rotter; en tanto que en subsitios de $1 \mathrm{~m}^{2}$ se recolectó la vegetación herbácea, hojarasca y humus. El manejo de la biomasa se realizó igual que la descrita para obtener la FC de cada componente. La biomasa de los árboles sombra se estimó con las ecuaciones alométricas de la Tabla 1.

\section{Análisis de datos}

Se realizaron las pruebas de normalidad y homogeneidad de varianzas con la prueba de Shapiro Wilk y Bartlett; las variables biomasa de cafetales, herbáceas y hojas que no cumplieron los supuestos de normalidad y homogeneidad de varianzas se transformaron con la función $\log (x)$, mientras que la biomasa de árboles sombra se transformó con $\sqrt{x}$. 
Tabla 1. Ecuaciones utilizadas para determinar la biomasa de árboles sombra.

\begin{tabular}{cccc}
\hline Especie & Ecuación de biomasa & $\mathrm{R}^{2}$ & Fuente \\
\hline Citrus sinensis L. (Osbeck) & $Y=6.64+0.279 x(A B)+0.000514 x(A B)^{2}$ & 0.94 & Espinoza-Domínguez et al. 2012 \\
Inga spp & $\ln Y=-1.76+(2.26 x \operatorname{Ln}(D N))$ & 0.97 & \\
Alnus glabrata F. & $\ln Y=-2.14+(2.23 x \operatorname{Ln}(D N))$ & 0.97 & Acosta-Mireles et al. 2002 \\
Clethra hartwegii & $\ln Y=-1.90+(2.15 x \operatorname{Ln}(D N))$ & 0.99 & \\
Liquidambar macrophylla Oerst. & $\ln Y=-2.22+(2.26 x \operatorname{Ln}(D N))$ & 0.99 & \multirow{2}{*}{ Chávez-Pascual et al. 2013 } \\
Pinus chiapensis Mart. And. & $\ln Y=0.236255 x N^{1.97173} x H T^{0.48104}$ & 0.95 & logaritmo natural.
\end{tabular}

Los datos se sometieron a un análisis de varianza y pruebas de separación de medias (Duncan, 0.05). La estructura del SAFC se describió mediante análisis de frecuencias por categorías diamétricas en intervalos de $5 \mathrm{~cm}$ y categorías de altura de $5 \mathrm{~m}$; también se realizaron pruebas de bondad de ajuste con la prueba de Ji-Cuadrada $(\alpha=0.05)$ para evaluar independencia entre especies y categorías estructurales. Para determinar el modelo con mejor bondad de ajuste se seleccionaron las variables con mayor significancia para la predicción de biomasa por compartimiento de los cafetos, mediante el procedimiento STEPWISE; los análisis de regresión se realizaron con los procedimientos REG y NLIN para seleccionar las ecuaciones alométricas con mayor bondad de ajuste. Todos los análisis se realizaron con el paquete estadístico Statistical Analysis System.

\section{RESULTADOS}

\section{Estructura del sistema agroforestal}

El sistema agroforestal de café evaluado se caracteriza por una estructura vertical y horizontal irregular (Figura 1). La prueba de independencia, establece una alta relación $(p<0.0001)$ entre las categorías de diámetro y altura de las especies arbóreas con base en su densidad por hectárea.

Las variedades sembradas de $C$. arabica son typica $(79 \%)$, caturra amarillo $(12.5 \%)$, mondonovo $(8.0 \%)$ y caturra rojo $(0.5 \%)$. Los árboles sombra representan el $12 \%$ del total con 14 especies de 13 géneros y familias. Las especies con mayor densidad son Inga edulis Mart. (749 árboles ha ${ }^{-1}$ ), la cual se encuentra distribuida en las categorías de diámetros de 5 a $45 \mathrm{~cm}$ y alturas de 5 a $20 \mathrm{~m}$, y Liquidambar styraciflua L. (493 árboles ha ${ }^{-1}$ ). Otras especies arbóreas son Rapanea myricoides Schltdl., Fraxinus uhdei (Wenz.) Lingelsh, Clethra hartwegii Britt, Alcornea latifolia L. con menos de 75 árboles ha ${ }^{-1}$, que proporcionan sombra al café, y leña. Algunas especies de importancia económica, por la cosecha de sus frutos, en el estrato inferior junto a $C$. arabica son Persea americana Mill (175 árboles ha ${ }^{-1}$ ), Citrus sinensis L. (Osbeck), Eriobotrya japonica (Thunb.) Lindl, Cecropia obtusifolia Bertol., Alnus glabrata Fernald, Heliocarpus donnellsmithii Rose, Pinus chiapensis (Martínez) Andresen y Citrus aurantifolia (Christm.) Swingle (25 árboles ha ${ }^{-1}$ ).

\section{Acumulación de biomasa aérea}

La cobertura de copa $(\mathrm{CoC})$ con base en la densidad de árboles sombra tuvo efectos significativos en la acumulación de biomasa total en los sitios $(p<0.04)$; los sitios con $\mathrm{CoC}$ de nivel medio tuvieron la mayor acumulación de biomasa aérea total y variabilidad $\left(87.02 \pm 33.3 \mathrm{t} \mathrm{ha}^{-1}\right)$, lo que se ve influenciado por el mayor contenido biomasa en árboles sombra $\left(78.4 \pm 29.4 \mathrm{t} \mathrm{ha}^{-1}\right)$. Mientras que los sitios con cobertura de $\mathrm{CoC}$ baja presentaron la menor acumulación de biomasa total (34.75 \pm $16.99 \mathrm{t} \mathrm{ha}^{-1}$ ). Sin importar el nivel de cobertura, la mayor proporción de la biomasa total en los sitios se encontró en los árboles sombra. En los sitios con nivel de cobertura de copa medio la biomasa acumulada en los árboles sombra fue de $78.4 \mathrm{t} \mathrm{ha}^{-1}$, valor que es superior estadísticamente a los valores de 17.89 y $29.8 \mathrm{t} \mathrm{ha}^{-1}$ que acumularon los árboles sombra de los sitios con coberturas de sombra baja y alta (Tabla 2). 


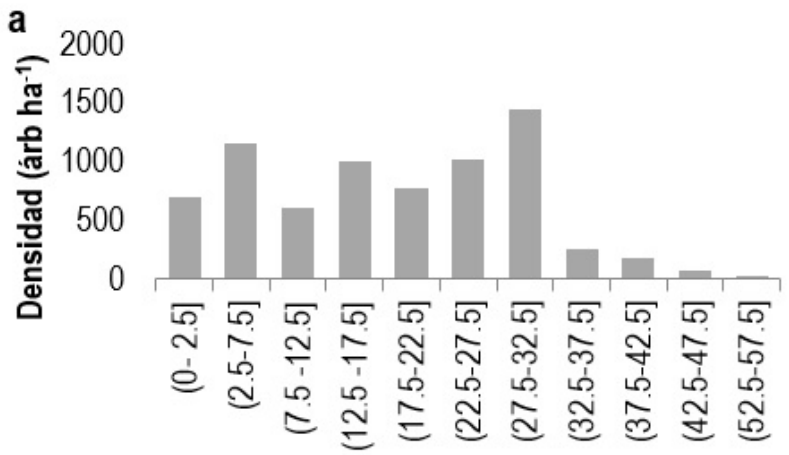

Categoría diamétrica $(\mathrm{cm})$

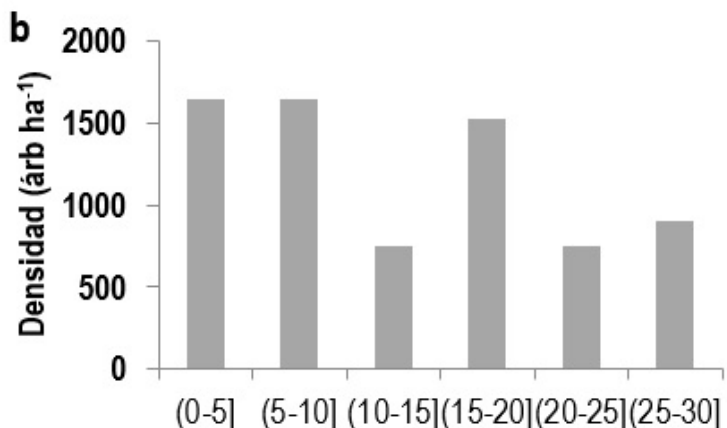

Categoría de altura $(\mathrm{m})$

Figura 1. Estructura horizontal (a) y vertical (b) del sistema agroforestal de café.

Tabla 2. Contenido de biomasa por componente de sistema agroforestal ( $t \mathrm{ha}^{-1}$ ) en función del tratamiento.

\begin{tabular}{|c|c|c|c|}
\hline \multirow{2}{*}{ Componente/ (significancia) } & \multicolumn{3}{|c|}{ Tratamiento (porcentaje de cobertura de copa) } \\
\hline & Bajo $(\leq 43)$ & Media $(43-86)$ & Alta (86-129) \\
\hline $\begin{array}{l}\text { Cafetos } \\
(0.0764)\end{array}$ & $\begin{array}{l}4.75 \pm 2.9^{a} \\
(13.6)\end{array}$ & $\begin{array}{l}1.21 \pm 0.79^{a} \\
(1.4)\end{array}$ & $\begin{array}{l}1.45 \pm 0.17^{a} \\
(3.3)\end{array}$ \\
\hline $\begin{array}{l}\text { Árboles sombra } \\
(0.0433)\end{array}$ & $\begin{array}{l}17.89 \\
(51.5)\end{array}$ & $\begin{array}{l}78.4 \pm 29.4^{a} \\
(90.1)\end{array}$ & $\begin{array}{l}29.8 \pm 13.3^{b} \\
(68.7)\end{array}$ \\
\hline $\begin{array}{l}\text { Necromasa } \\
(0.0720)\end{array}$ & $\begin{array}{l}1.55 \pm 0.43^{a b} \\
(4.5)\end{array}$ & $\begin{array}{l}1.96 \pm 0.34^{a} \\
(2.25)\end{array}$ & $\begin{array}{l}1.0 \pm 0.43^{b} \\
(2.3)\end{array}$ \\
\hline $\begin{array}{l}\text { Herbáceas } \\
(0.2647)\end{array}$ & $\begin{array}{l}1.56 \pm 0.65^{a} \\
(4.5)\end{array}$ & $\begin{array}{l}0.56 \pm 0.19^{a} \\
(0.64)\end{array}$ & $\begin{array}{l}1.26 \pm 1.26^{a} \\
(2.9)\end{array}$ \\
\hline $\begin{array}{l}\text { Hojarasca } \\
(0.5572)\end{array}$ & $\begin{array}{l}1.23 \pm 0.8^{a} \\
(3.5)\end{array}$ & $\begin{array}{l}1.93 \pm 1.89^{a} \\
(2.21)\end{array}$ & $\begin{array}{l}0.75 \pm 0.19^{a} \\
(1.7)\end{array}$ \\
\hline $\begin{array}{l}\text { Humus } \\
(0.1378)\end{array}$ & $\begin{array}{l}7.73 \pm 1.69^{a} \\
(22.2)\end{array}$ & $\begin{array}{l}2.86 \pm 2.5^{a} \\
(3.3)\end{array}$ & $\begin{array}{l}9.13 \pm 1.69^{a} \\
(21.0)\end{array}$ \\
\hline Total $(0.0752)$ & $34.75 \pm 16.99^{b}$ & $87.02 \pm 33.3^{a}$ & $43.47 \pm 13.1^{a b}$ \\
\hline
\end{tabular}

Se presenta la media $\left(\mathrm{t} \mathrm{ha}^{-1}\right) \pm$ la desviación estándar y entre paréntesis se presenta la proporción de cada compartimiento por tratamiento). En cada fila, medias con letras iguales no muestran diferencias significativas (Duncan, $\alpha=0.05$ ) y debajo de cada componente se presenta su significancia $(\mathrm{Pr}>\mathrm{F})$.

\section{Modelos de biomasa para cafetos}

La biomasa en tallo y total aéreo se estima con una bondad de ajuste de 0.89 y 0.88 , respectivamente; con el diámetro al pie y la altura de cafetos en una función lineal cuadrática. El intercepto de ambos modelos presentó significancia $(\mathrm{p}<$ 0.0001 ), pero no la altura asociada a la pendiente $(p=0.05)$; aunque ésta última es importante involucrar para estimar más de $88 \%$ de la variación en la biomasa de los cafetos (Tabla 3). La biomasa en ramas se ajustó con las variables diámetro al pie y altura, con una ecuación no lineal, múltiple con significancia $\mathrm{p}=0.0001$ y bondad de ajuste de 0.71 .
Para la biomasa en hojas se utilizó una ecuación exponencial con significancia $p=0.0001$ y bondad de ajuste de 0.75 .

\section{DISCUSIÓN}

\section{Estructura del sistema agroforestal}

Las estructuras horizontal y vertical muestran una distribución irregular teniendo una alta dependencia $(p<0.0001)$ entre las categorías diamétrica y de altura con base en la densidad. Verticalmente se puede apreciar el estrato arbóreo cuya función principal es regular las condiciones microclimáticas 
Tabla 3. Valores de ajuste de los modelos de predicción de biomasa para compartimentos aéreos de café.

\begin{tabular}{|c|c|c|c|c|c|c|c|}
\hline Variable & Parámetro & Estimación & Error estándar & $\operatorname{Pr}>\mathrm{F}$ & $\mathrm{R}^{2}$ & $\mathrm{R}^{2}$ aj. & CME \\
\hline Biomasa (tallo) $=\beta 0 d b^{2} x \beta 1 a t$ & & & & 0.0001 & 0.89 & & 0.74805 \\
\hline $\mathrm{db}^{2}$ & b0 & 0.0581 & 0.0101 & 0.0001 & & & \\
\hline At & b1 & 0.1003 & 0.10126 & 0.3304 & & & \\
\hline $\operatorname{Biomasa}\left(\right.$ tallo) $=\beta 0 d b^{2} x \beta 1 a t$ & & & & 0.0001 & 0.88 & & 1.41896 \\
\hline $\mathrm{db}^{2}$ & b0 & 0.0774 & 0.01392 & 0.0001 & & & \\
\hline At & b1 & 0.0880 & 0.13946 & 0.5329 & & & \\
\hline Biomasa (ramas) $=\beta 0 d b^{\beta 1} x a t^{\beta 2}$ & & & & 0.0001 & & 0.71 & 0.1567 \\
\hline Intercepto & b0 & 0.0043 & 0.00461 & & & & \\
\hline $\mathrm{Db}$ & b1 & 2.3367 & 0.6217 & & & & \\
\hline At & b2 & 0.4521 & 0.7296 & & & & \\
\hline Biomasa (hojas) $=e^{\beta 1(\beta 2 d b-3 a t)}$ & & & & 0.0001 & & 0.75 & 0.00275 \\
\hline Intercepto & b0 & -4.4537 & 0.533 & & & & \\
\hline $\mathrm{Db}$ & b1 & 0.3415 & 0.0758 & & & & \\
\hline At & b2 & -0.0442 & 0.1693 & & & & \\
\hline
\end{tabular}

en los cafetos del nivel inferior (Siles et al. 2010), lo que permite una mayor estabilidad y resiliencia del SAFC (Craparo et al. 2015).

La mayor densidad de 1650 árboles ha ${ }^{-1}$ en la categoría de altura menor a $10 \mathrm{~m}$ propicia mayor competencia por nutrientes y luz en los cafetos, lo que reduce el rendimiento, por lo que se recomienda realizar aclareos en los árboles sombra en esta categoría de altura. De manera horizontal se tiene una sobre posición de las copas, lo que ayuda a disminuir la pérdida de suelo por erosión hídrica (Pérez et al. 2012) y reduce la evapotranspiración del suelo y del café (Lin 2010). De manera negativa la excesiva cobertura influye en la proliferación de plagas y enfermedades como Hypothenemus hampei, Hemileia vastatrixy y Mycenaci tricolor (Maldonado-Martínez et al. 2014).

La especie con mayor densidad fue $I$. edulis (749 árboles ha ${ }^{-1}$ ), que pertenece a la familia Fagácea y se caracteriza por alta fijación de nitrógeno benéfico para los cafetos (Hergoualc'h et al. 2012). Otra especie importante característica del bosque mesofilo de montaña es $L$. styraciflua., con mayor frecuencia en el estrato dominante $(25$ - $30 \mathrm{~m}$ ) y 493 árboles ha $^{-1}$ (Acosta-Mireles et al. 2002).

EI SAFC utiliza la sombra de árboles del bosque nativo (mesófilo de montaña), de forma similar a lo reportado por García-de la Cruz et al. (2012) quienes indican que intercalando especies introducidas (frutales), se produce una estructura compleja con árboles sombra entre 20 y $30 \mathrm{~m}$ de altura, los cuales se aprovechan como alimento, leña, medicinales, entre otros usos; además de que no se utilizan insumos externos para fertilizar, por lo que se considera un policultivo tradicional (Moguel y Toledo 1999). Las especies encontradas son las mismas que se reportan para el SAFC de la región, como los géneros Inga y Persea (Hernández-Vásquez et al. 2012, Bolaños y González 2013); identificando 14 especies, cuatro menos que las reportadas por Asase y Tetteh (2010) para un SAFC.

\section{Acumulación de biomasa aérea}

Los árboles sombra tuvieron alta significancia ( $p<0.04)$, ya que es el compartimento que almacena la mayor cantidad de biomasa en todo el SAFC, además presentó el mayor efecto por tratamiento de $\mathrm{CoC}$. Siendo el nivel medio de tratamiento de $\mathrm{CoC}$ el de mayor biomasa acumulada con $87.02 \mathrm{t} \mathrm{ha}^{-1}$, valor que es menor al obtenido por Magaña et al. (2004) y Espinoza-Domínguez et al. (2012) con 100 y $102 \mathrm{t} \mathrm{C} \mathrm{ha}^{-1}$.

En el tratamiento medio se tiene la mayor acumulación de biomasa en árboles sombra $78.4 \mathrm{t}$ $\mathrm{ha}^{-1}$ disminuyó la presencia de herbáceas a $0.56 \mathrm{t}$ $\mathrm{ha}^{-1}$, pero en el tratamiento bajo se tuvo la menor acumulación de biomasa arbórea con $17.89 \mathrm{t} \mathrm{ha}^{-1}$ y la mayor presencia de herbáceas $\left(1.56 \mathrm{t} \mathrm{ha}^{-1}\right)$, lo que coincide con Moraga et al. (2011). En el 
nivel bajo de $\mathrm{CoC}$ los árboles sombra acumularon menos biomasa (17.89 $\mathrm{t} \mathrm{ha}^{-1}$ ), pero debido al espaciamiento que dejan los árboles sombra, los cafetos lograron mayor desarrollo con $4.75 \mathrm{t} \mathrm{ha}^{-1} \mathrm{de}$ biomasa, valor que es mayor al obtenido por Moraga et al. (2011) en cafetales a pleno sol.

Para la necromasa se tuvo la mayor acumulación en el SAF con CoC de nivel medio (43 a 86\%, Tabla 2), lo que coincide con Noponen et al. (2013), y Maldonado-Martínez et al. (2014). Pero el valor es menor al encontrado por Cerda et al. (2013) de $6.3 \mathrm{t} \mathrm{ha}^{-1}$ en un SAF de cacao. La biomasa del humus (mantillo) osciló entre 2.86 y $7.73 \mathrm{t} \mathrm{ha}^{-1}$, pero no se observaron diferencias significativas por efecto de la $\mathrm{CoC}$, valores similares fueron reportados por Álvarez-Arteaga et al. (2013) para SAF de la Sierra Norte de Oaxaca. Al respecto se sabe que la acumulación de biomasa en el SAFC aporta nutrientes al suelo (Romero-Alvarado et al. 2002); conserva la biodiversidad (Valencia et al. 2014), sin afectar la producción de café (Romero-Alvarado et al. 2002). Considerando en promedio de todos los tratamientos la sumatoria de la biomasa aérea (55.08 $\mathrm{t} \mathrm{ha}^{-1}$ ) se tiene una similitud con Cerda et al. (2013) que fue de $50 \mathrm{t} \mathrm{ha}^{-1}$ en sistemas agroforestales de cacao.

\section{Modelos de biomasa para cafetos}

Las ecuaciones alométricas permiten estimar biomasa o carbono de manera indirecta, con variables de fácil medición en campo para determinar la biomasa o carbono en SAFC (EspinozaDomínguez et al. 2012, Ehrenbergerová et al. 2015). De manera directa mediante muestreo destructivo Chave et al. (2005) ajustaron ecuaciones para un bosque, Acosta-Mireles et al. (2002) para un bosque mesófilo y Segura et al. (2006) para un SAFC. Para el ajuste de ecuaciones se utilizó el diámetro al pie $(\mathrm{p} \leq 0.0001)$ y la altura, lo que difiere con estudios realizados para Quercus magnoliaefolia Née, especies leñosas neotropicales, el SAF de Tea y paisajes agrícolas, en los que se utilizó el diámetro a la altura del pecho (Kuyah et al. 2012, Douterlungne et al. 2013, Kalita et al. 2015). En ecuaciones para $C$. arabica, se ha utilizado el diámetro a $40 \mathrm{~cm}$ (Negash et al. 2013), el diámetro normal (Hairiah et al. 2001) y el diámetro a $15 \mathrm{~cm}$ de la base (Segura et al. 2006), reportándose que el modelo tuvo mayor ajuste con la altura. Mientras que Rutishauser et al. (2013), afirman que la altura puede reducir la incertidumbre para predecir biomasa. Al incluir la altura del cafeto y el diámetro al pie, el coeficiente de determinación para la ecuación lineal fue superior $\left(R^{2}=0.87\right)$ al reportado por Segura et al. (2006), quienes utilizaron el diámetro a $15 \mathrm{~cm}$ del suelo, mientras que Negash et al. (2013) reportan que el mejor ajuste se tiene con el diámetro a $40 \mathrm{~cm}$ del suelo. La alta variabilidad en los datos encontrados podría ser por efecto de Hemileia vastatrix, que afecta el crecimiento de los cafetos (Honorato et al. 2015).

\section{CONCLUSIONES}

El sistema agroforestal de café orgánico se caracteriza por tener estructuras verticales y horizontales irregulares, debido a las diversas especies que proporcionan sombra al sistema agroforestal. Se registraron 78 árboles que se agrupan en 14 especies arbóreas de 13 familias que promueven una serie de sinergias entre árboles sombra y cafetales. La $\mathrm{CoC}$ de nivel medio fue la que presentó mayor efecto por tratamiento, generando la mayor acumulación de biomasa en árboles sombra, necromasa, hojarasca y en la sumatoria de todos los compartimientos, presentano los árboles sombra diferencias significativas por efecto de tratamiento, con acumulación promedio del $70 \%$ de la biomasa total. El ajuste de ecuaciones, que estiman biomasa en cafetos, se generó a partir de las variables diámetro al pie y la altura por compartimiento, las cuales tuvieron la mayor correlación con la biomasa.

\section{LITERATURA CITADA}

Acosta-Mireles MJ, Vargas-Hernández J, Velázquez-Martínez A, Etchevers-Barra JD (2002) Estimación de la biomasa aérea mediante el uso de relaciones alométricas en seis especies arboreas en Oaxaca, México. 
Agrociencia 36: 725-736.

Álvarez-Arteaga G, Calderón NE, Krasilnikov P, García-Oliva F (2013) Almacenes de carbono en bosques montaños de niebla de la Sierra Norte de Oaxaca, México. Agrociencia 47: 171-80.

Andrade HJ, Marín LM, Pachón DP (2014) Fijación de carbono y porcentaje de sombra en sistemas de producción de café (Coffea arabica L.) en el Líbano, Tolima, Colombia. Bioagro 26: 127-132.

Asase A, Tetteh DA (2010) The role of complex agroforestry systems in the conservation of forest tree diversity and structure in southeastern Ghana. Agroforestry Systems 79: 355-368.

Benjamín-Ordoñez J, Masera O (2001) Captura de carbono ante el ambio climático. Madera y Bosques 7: 3-12.

Bolaños M, González RA (2013) Café orgánico de sombra en el Rincón de Ixtlán, Oaxaca, México. Agroforestería Ecológica 53: 1689-1699.

Cerda BR, Espin CT, Cifuentes M (2013) Carbono en sistemas agroforestales de cacao de la Reserva Indígena Bribri de Talamanca, Costa Rica. Agroforestería en las Américas 49: 33-41.

Craparo ACW, Van PJA, Läderach P, Jassogne LTP, Grab SW (2015) Coffea arabica yields decline in tanzania due to climate change: global implications. Agricultural and Forest Meteorology 207: 1-10.

Chave J, Andalo C, Brown S, Cairns MA, Chambers JQ, Eamus D, et al. (2005) Tree allometry and improved estimation of carbon stocks and balance in tropical forests. Oecologia 145: 87-99.

Chávez-Pascual EY, Rodriguez-Ortiz G, Carrillo-Rodriguez JC, Enriquez-del Valle JR, Chávez-Servia JL, Campos-Ángeles JV (2013) Factores de expansión de biomasa aérea para Pinus chiapensis (Mart.) Andersen. Revista Mexicana de Ciencias Agricolas 6: 1273-84.

Douterlungne D, Herrera-Gorocica AM, Ferguson BG, Siddique I, Soto-Pinto L (2013) Ecuaciones alométricas para estimar biomasa y carbono de cuatro especies leñosas neotropicales con potencial para la restauración. Agrociencia 47: 385-97.

Ehrenbergerová L, Cienciala E, Kučera A, Guy L, Habrová H (2015) Carbon stock in agroforestry coffee plantations with different shade trees in Villa Rica, Peru. Agroforestry Systems 90: 433-45.

Escamilla PE, Licona PAL, Díaz CS Santoyo CHV, Sosa R, Rodríguez RL (1997) Los sistemas de producción del café en el centro de Veracruz, México. Un análisis tecnológico. Revista de Historia 30: 41-67.

Espinoza-Domínguez W, Krishnamurthy L, Vázquez-Alarcón A, Torres- Rivera A (2012) Almacén de carbono en sistemas agroforestales con café. Revistas Chapingo Serie Ciencias Forestales y del Ambiente 18: 57-70.

García-de la Cruz L, Olivares-López A, Ramos-Prado JM (2012) Estructura y composición arbórea de un fragmento de bosque mesófilo de montaña en el estado de Veracruz. Revista Chapingo Serie Ciencias Forestales y del Ambiente 19: 91-101

García MLE, Valdéz HJI, Luna CM, López MR (2015) Estructura y diversidad arbórea en sistemas agroforestales de café en la sierra de Atoyac, Veracruz. Madera y Bosques 21: 69-82.

Häger A (2012) The effects of management and plant diversity on carbon storage in coffee agroforestry Systems in Costa Rica. Agroforestry systems 86: 159-174.

Hairiah K, Sitompul S, Van NM, Palm CA (2001) Carbon stocks of tropical land use systems as part of the global carbon balance: effects of forest conversion and options for clean development activities. International Centre for Research in Agroforestry. Bogor, Indonesia. 49 p. 
Hergoualc'h K, Blanchart E, Skiba U, Hénault C, Harmand JM (2012) Changes in carbon stock and greenhouse gas balance in a coffee (Coffea arabica) monoculture versus an agroforestry system with Inga densiflora, in Costa Rica. Agriculture, Ecosystems and Environment 148: 102-10.

Hernández-Vásquez E, GV Campos-Ángeles, JR Enríquez-Del Valle, G Rodríguez-Ortiz, VA Velasco-Velasco (2012). Captura de carbono por Inga jinicuil schltdl. En un sistema agroforestal de café bajo sombra. Revista Mexicana de Ciencias Forestales 3: 11-21.

Honorato JJ, Zambolim L, Aucique-Pérez CE, Resende RS, Rodrigues FA (2015) Photosynthetic and antioxidative alterations in coffee leaves caused by epoxiconazole and pyraclostrobin sprays and Hemileia vastatrix infection. Pesticide Biochemistry and Physiology 123: 31-39.

INEGI (2006) Prontuario de información geográfica municipal de los Estados Unidos Mexicanos. San Juan Juquila Vijanos, Oaxaca Clave geoestadística 202001. Instituto Nacional de Estadística y Geografía. http://www3.inegi.org.mx/contenidos/app/mexicocifras/datos_geograficos/20/20201.pdf. 8. Fecha de consulta 3 de abril de 2017.

IPCC (2007) Climate change 2007: The physical science basis. Contribution of Working Group I to the fourth assessment report of the intergovernmental panel on climate change. Solomon S, Qin D, Manning M, Chen Z, Marquis M, Averyt KB, Tignor M, Miller HL (Eds). Cambridge University Press. United Kingdom and New York. 996 p.

Kalita RM, Kumar DA, Jyoti NA (2015) Allometric equuations for estimating above-and belowground biomass in Tea (Camellia sinensis (L.) O. Kuntze) agroforestry system of Barak Valley, Assam, northeast India. Biomass \& Bioenergy 83: 42-49.

Kuyah S, DietzJ, Muthuri C, JamnadassR, Mwangi P, Coe R, et al. (2012) Allometric equations for estimating biomass in agricultural landscapes: I. Abovegroud biomass. Agriculture, Ecosystems and Environment 158: $216-224$.

Lin BB (2010) The role of agroforestry in reducing water loss through soil evaporation and crop transpiration in coffee agroecosystems. Agricultural and Forest Meteorology 150: 510-518.

Magaña SM, Harmand JM, Hergoualc'h K (2004) Cuantificación del carbono almacenado en la biomasa aérea y el mantillo en sistemas agroforestales de café en el suroeste de Costa Rica. Agroforestería en las Americas 41: 98-104.

Maldonado-Martínez R, Rodríguez-Ortiz G, Enríquez-Del Valle JR, Carrillo-Rodríguez JC, Pérez-León MI (2014) Efecto de la cobertura arbórea en sistemas de café orgánico en el sur de Oaxaca. Revista Mexicana de Agroecosistemas 1: 12-19.

Moguel P, Toledo VM (1999) Biodiversity conservation in tradicional coffee systems of México. Conservation Biology 13: 11-21

Montagnini F, Somarriba E, Murgueitio E, Fasola H, Eibl B (2015) Sistemas agroforestales funciones productivas, socioeconómicas y ambientales. Serie técnica. Informe técnico 402. CATIE, Costa Rica. Edit CIPAV, Colombia. 454 p.

Moraga P, Bolaños R, Pilz M, Munguía R, Jurgen A, Barios M, et al (2011) Árboles de sombra e intensidad del cultivo afectan al rendimiento de café (Coffea arabica L.) y la valoración ecológica en Masatepe, Nicaragua. La Calera 11: 41-47.

Negash M, Starr M, Kanninen M, Berhe L (2013) Allometric equations for estimating aboveground biomass of Coffea arabica L. grown in the Rift Valley escarpment of Ethiopia. Agroforestry Systems 87: 953-966. 
Noponen MRA, Healey JR, Soto G, Haggar JP (2013) Sink or source-the potential of coffee agroforestry systems to sequester atmospheric $\mathrm{CO} 2$ into soil organic carbon. Agriculture, Ecosystems and Environment 175: $60-68$.

Pérez NJ, Valdés VE, Ordaz CV (2012) Cobertura vegetal y erosión del suelo en sistemas agroforestales de café bajo sombra. Terra Latinoamericana 30: 249-59.

Ramachandran NPK (2011) Agroforestry systems and environmental quality: introduction. Journal of Environmental Quality 40: 784-790.

Ramachandran NPK, Nair VD (2014) Solid-fluid-gas: The state of knowledge on carbon-sequestration potential of agroforestry systems in africa. Current Opinion in Environmental Sustainability 6: 22-27.

Romero-Alvarado Y, Soto-Pinto L, García-Barrios L, Barrera-Gaytán JF (2002) Coffee yields and soil nutrients under the shades of Inga sp. vs. multiple species in Chiapas, Mexico. Agroforestry Systems 54: 215-224.

Rutishauser E, Noor'an F, Laumonier Y, Halperin J, Hergoualc'h R, Verchot KL (2013) Generic allometric model including height best estimate forest biomass and carbon stocks in Indonesia. Forest Ecology and Management 307: 219-225.

Santos MFE (2012) Plan municipal de desarrollo rural sustentable. Consejo Municipal de Desarrollo Rural Sustentable. 92 p.

Segura M, Kanninen M, Suárez D (2006) Allometric models for estimating aboveground biomass of shade trees and coffee bushes grown together. Agroforestry Systems 68: 143-50.

Siles P, Harmand JM, Vaast P (2010) Effects of inga densiflora on the microclimate of coffee (Coffea arabica L.) and overall biomass under optimal growing conditions in Costa Rica. Agroforestry Systems 78: 269-86.

Soto-Pinto L, Anzueto MMJ, Mendoza VJ, Jiménez FG, de Jong BHJ (2009) Carbon sequestration through agroforestry in indigenous communities of Chiapas, Mexico. Agroforestry Systems 78: 39-51.

Valencia V, García-Barrios L, West P, Sterling EJ, Naeem S (2014) The role of coffee agroforestry in the conservation of tree diversity and community composition of native forests in a biosphere reserve. Agriculture, Ecosystems and Environment 189: 154-63. 